\title{
Microscopy and Elemental Analysis on a Haynes-25 Crushed Weld Ring
}

Angelique D Wall ${ }^{1}$, Terry G. Holesinger ${ }^{1}$, Joseph P.M. Romero ${ }^{1}$, Ted F. Cousins ${ }^{2}$, William L. Stellwag Jr. $^{2}$, Joshua Phillips ${ }^{3}$ and Andrew Sandford ${ }^{3}$

1. Material Science Division, MST-16, Los Alamos National Laboratory, Los Alamos NM, USA.

2. Actinide Material Processing, AMMP-2, Los Alamos National Laboratory, Los Alamos NM, USA.

3. Sandia National Laboratory, Albuquerque NM, USA.

Scanning electron microscopy (SEM) with elemental analysis was used to characterize the metallurgical condition of heat source capsules. Heat sources are typically used for deep space missions where solar power doesn't provide enough power to maintain the electronics. Heat sources have been used for Radioisotope Thermoelectric Generators (RTG) for the Cassini mission and recent missions to Mars [1]. The heat generating component within the capsules is ${ }^{238} \mathrm{PuO}_{2}$ in the form of granules. The ${ }^{238} \mathrm{PuO}_{2}$ granules are typically triple encapsulated. For this study, only the analysis of innermost layer that had been in direct contact with the granules is discussed.

The metal selected for heat source containment has traditionally been T-111, an alloy of Tantalum, Tungsten, and Hafnium, due to the emission of radiation and the highly corrosive nature or $\mathrm{PuO}_{2}$. However, previous studies have shown that T-111 is subject to oxygen embrittlement [2][3]. Hence, other alloys are now under investigation as possible replacements for T-111. The current work is focused on results regarding Haynes-25, an alloy of Cobalt, Chromium, Nickel, and Tungsten. Initial work using $\mathrm{CeO}_{2}$ powder as a simulant was found to cause a similar oxygen embrittlement effect to that observed with the T-111 alloy. The process by which this occurs is believed to involve adsorbed water, coupled with a reaction/evaporation and condensation mechanism. In order to determine the viability of this material for ${ }^{238} \mathrm{PuO}_{2}$, granules were encapsulated, welded with an autogenous gas tungsten arc (GTA), and then the capsules contents were removed to isolate the "exposed" liner to test and characterize it. Since the weld area is typically the weakest part of the capsules, a crush test was performed on the weld.

Confined crush tests were conducted by removing rings using a slow speed Isomet diamond saw near the edge of the weld at a width of 0.125 inches $(3.175 \mathrm{~mm})$, see Figure 1 . The rings were ground using 400 grit sanding paper to improve inspections of samples for tears and cracks. The samples were crushed using a fixture in a vise to a height of approximately 0.130 inches $(3.3 \mathrm{~mm})$. No visual cracking was observed on any inner, outer, or edge surface. The crushed ring was then analyzed using a Hitachi S3500N Scanning Electron Microscope (SEM) to determine if there were any cracks or other observable damage to the crushed ring. The crushed ring was mounted on an aluminum stub using carbon tape with one of the cut sides facing up. After analysis on one side of the cut the crushed ring was rotated to image the second side, see Figure 2 a). SEM analysis confirmed that there were no cracks on the crushed rings. Secondary (SEI) and Back Scattered Electron Images (BSEI) were taken on both sides of the weld ring, see Figure $2 \mathrm{~b}$ ) and c). Elemental analysis was also performed by energy dispersive spectroscopy (EDS) on the crushed rings using an Oxford Instruments X-Max 50 Silicon Drift Detector (SDD). No unexpected elements were found, but inspection of the weld region was made difficult by the geometry of the crushed ring. To further investigate, weld sections were cut out of rings and mounted in epoxy and polished for further analysis via metallography, SEM imaging and elemental analysis with the EDS system [4]. 
References:

[1] PD Richardson II et al., Acers Conference (2009).

[2] Richardson II et al., International Conference and expo on advanced Ceramics and Composites (2015).

[3] C Duty et al., American Nuclear Society Embedded Topical Meeting - 2005 Space Nuclear Conference (2005) p. 294.

[4] This work is funded by the U.S. Department of Energy Office. Los Alamos National Laboratory, an affirmative action equal opportunity employer, is operated by TRIAD National Security, LLC, for the National Nuclear Security Administration of the U.S. DOE under contract 89233218CNA000001.

a)

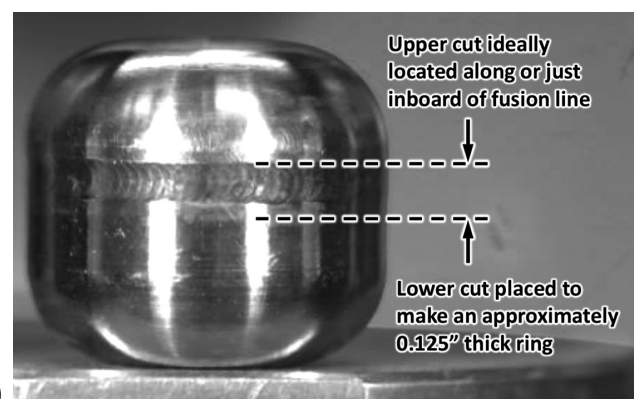

b)

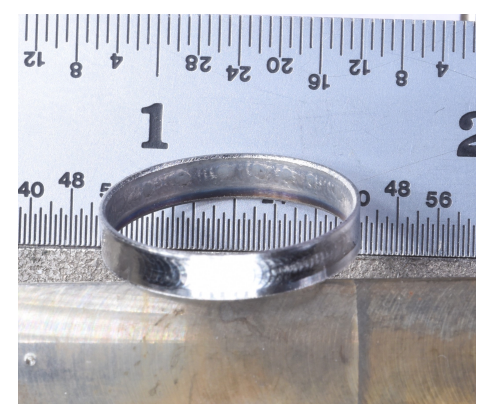

Figure 1. Optical images of (a) the fully assembled capsule and (b) a cross section through the capsule that contains the weld ring (images curtesy of Sandia National Laboratory).

a)

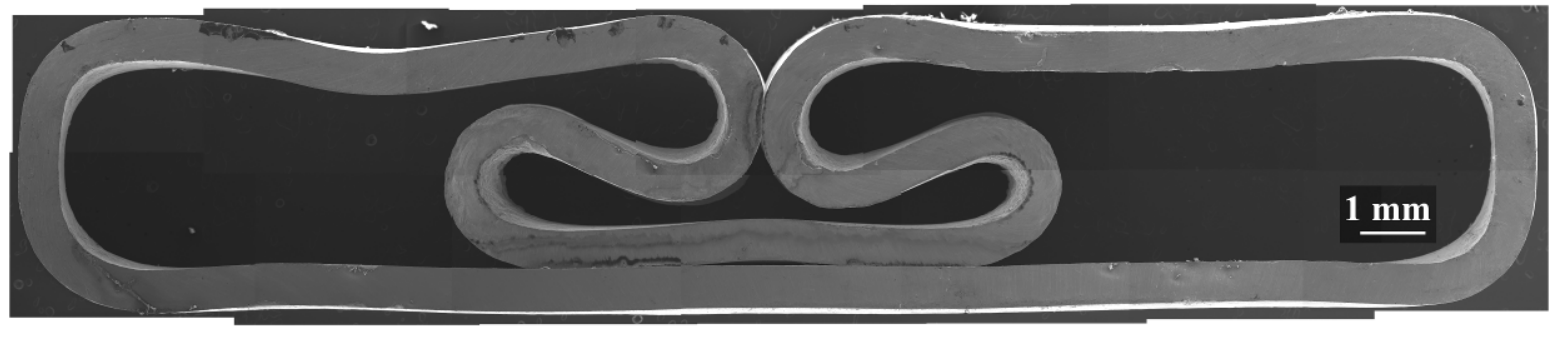

b)
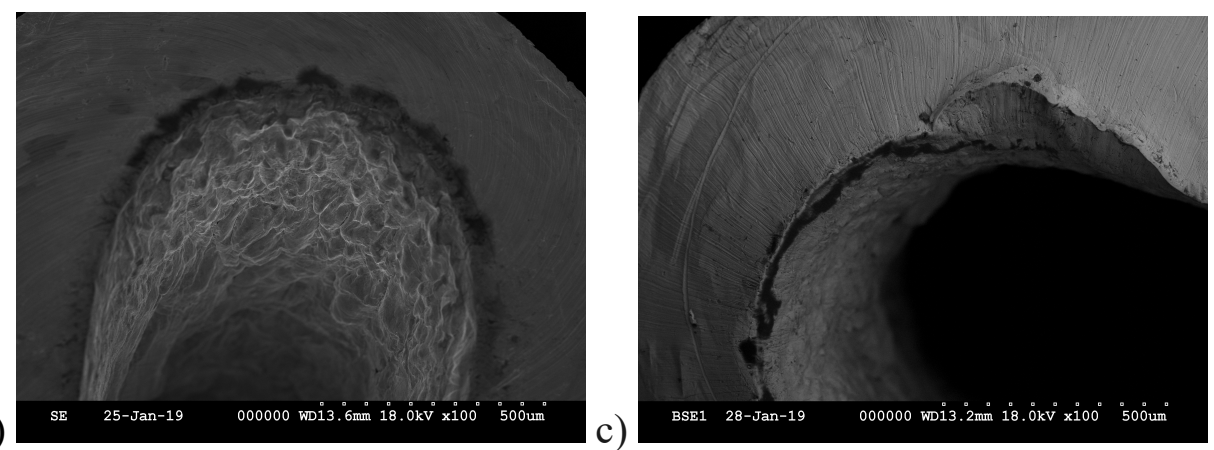

Figure 2. a) Crushed ring via stitched SEM images; b) Secondary Electron Image of the interior of one of the bends of the crushed ring. c) Back Scattered Electron Image of one of the interior of one of the bends of the crushed ring. 